\title{
Nano Sensor for Near Zero Emission Monitoring of Sulfur Hexafluoride
}

\section{Lijun Zhang*, Kecheng Liu, Hesong Han, Yanning Gao, Chongming Chen, Rongxue Shi}

Institute of Environmental Protection and Chemical Technology, State Grid Hebei Electric Power Research Institute, Shijiazhuang 050021, China

*to whom all correspondence should be addressed: e-mail: jlzhanglj@163.com

\section{CATALUMINESCENCE SENSOR}

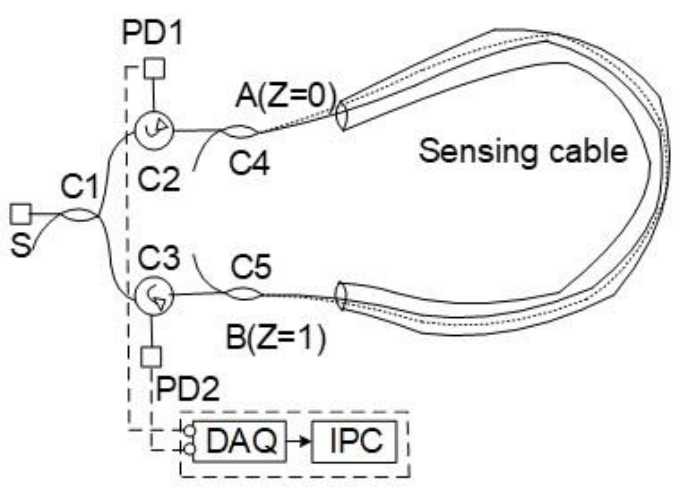

\section{NANOMATERIALS UNIT LAYOUT}

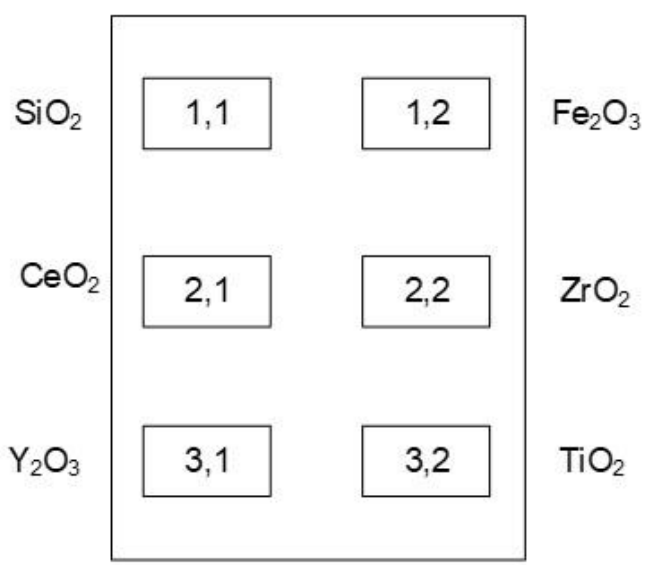

\section{Graphical Abstract}

\begin{abstract}
Aiming at the problems of long monitoring time, low sensitivity and low precision of traditional methods, a nano sensor for near zero emission monitoring of sulfur hexafluoride $\left(\mathrm{SF}_{6}\right)$ was proposed. The characteristics of $\mathrm{SF}_{6}$ gas were analyzed. The infrared spectrum of $\mathrm{SF}_{6}$ gas was obtained by spectral absorption method. The laser of Laixi series is used as the hardware of $\mathrm{SF}_{6}$ gas monitoring. The nano sensor array is designed, and the near zero emission state of $\mathrm{SF}_{6}$ is monitored by using the nano sensor node optimization method. The experimental results show that compared with the traditional method, the nano sensor method can shorten the monitoring time (less than 3.0 seconds), improve the monitoring sensitivity and accuracy. The results show that the method can monitor the air emission and environmental pollution accurately and timely.
\end{abstract}

Keywords: Nanosensor, Sulfur Hexafluoride, Near Zero Emission, Sensor Array, Node Optimization.

\section{Introduction}

With the rapid development of social economy, urban substation building area is becoming smaller and smaller, which requires that the floor area of distribution equipment needs to be further reduced. In order to ensure the insulation and arc extinguishing performance in a limited space, the use of $\mathrm{SF}_{6}$ equipment will be further promoted (Sekhar et al., 2020; Yamamura et al., 2019). The extensive use of $\mathrm{SF}_{6}$ equipment, on the one hand, makes power production more safe and reliable; on the other hand, $\mathrm{SF}_{6}$ gas is more and more likely to cause harm to the environment, equipment, especially the personal health and safety of on-site workers (Morscher and Maxwell, 2019). To ensure the normal operation of the equipment and protect the health and safety of onsite workers, effective means must be taken to monitor the $\mathrm{SF}_{6}$ gas in the equipment area (Mishra et al., 2019).

The research on the monitoring technology of $\mathrm{SF}_{6}$ in foreign countries starts earlier, and the development of monitoring construction is much more advanced than that in China. With the in-depth development of the 
research, the monitoring of $\mathrm{SF}_{6}$ zero emission has become a research hotspot in recent years (Blanco-Rayon et al., 2019). For many years, many researches have been carried out on the monitoring of $\mathrm{SF}_{6}$ leakage at home and abroad. The current $\mathrm{SF}_{6}$ leakage monitoring technologies at home and abroad are mainly classified into the following categories: ultraviolet imaging remote sensing monitoring, acoustic method, high insulation method, etc. Ultraviolet imaging remote sensing monitoring method has advantages of high detection accuracy, high practicability, high temporal resolution and spatial resolution of ultraviolet imaging. It can intuitively obtain the two-dimensional distribution of pollutant gas concentration and the emission rate, so it plays an important role in monitoring environmental pollution (Zhang et al., 2020). Based on the ultraviolet imaging detection technology, the gas concentration information is obtained, which verifies the great application prospect of this method in remote sensing monitoring of factory chimney and ship exhaust pollution emission. However, the monitoring time of this method is too long, which cannot meet the needs of real-time monitoring (Zuo et al., 2020). The principle of acoustic method is to monitor the sound by using the principle that the speed of sound propagation in $\mathrm{SF}_{6}$ gas is slower than that in the atmosphere. The 3-026-R002 $\mathrm{SF}_{6}$ gas alarm of DILO company in Germany can monitor the leakage of $\mathrm{SF}_{6}$ gas when the content of $\mathrm{SF}_{6}$ gas in the environment exceeds $2 \%$. The system can connect up to 6 monitoring points. The system is limited due to following main disadvantages, including low monitoring sensitivity, the exceeding the theoretical safety upper limit of $1000 \mathrm{ppm}\left(\mathrm{cm}^{3} / \mathrm{m}^{3}\right)$, and unsatisfactory monitoring accuracy. The high insulation method is a gas leak detector that uses the high insulation characteristics of $\mathrm{SF}_{6}$ gas and uses high-voltage corona discharge technology. The 3-033-R00 sulphur hexafluoride gas alarm from DILO in Germany and the TIF5650A/TIF5750A qualitative $\mathrm{SF}_{6}$ gas leak detector from TIF in the United States can qualitatively monitor the leakage of $\mathrm{SF}_{6}$ in the environment. This type of instrument needs to be calibrated before use, but is not suitable for long-term real-time monitoring (Garmendia et al., 2019; Toma et al., 2019; Wilde, 2019).

Aiming at the problems of traditional monitoring methods, a nano sensor for near zero emission monitoring of sulfur hexafluoride was proposed (He et al., 2018a). The method of monitoring $\mathrm{SF}_{6}$ gas leakage with nano sensor designed in this paper has the advantages of low cost and strong practicability. It can quickly and effectively monitor $\mathrm{SF}_{6}$ gas leakage and the location of leakage point.

\section{EXPERIMENT}

\subsection{Characteristics of $\mathrm{SF}_{6}$ Gas}

$\mathrm{SF}_{6}$ has good electrical insulation performance and excellent arc extinguishing performance. It is a new generation of ultra-high voltage dielectric material superior to air and oil. Therefore, $\mathrm{SF}_{6}$ has been widely used in related equipment of power system (Marchant et al., 2019). $\mathrm{SF}_{6}$ gas leakage is not easy to be detected, and will seriously reduce the ability of gas insulation and arc extinguishing, bringing security risks to the operation of power equipment. The leakage of toxic decomposition products produced by $\mathrm{SF}_{6}$ during high voltage arc extinguishing will cause harm to personal safety (Grace and Manju, 2019). Soapy water or hand-held leak detector are normally adopted in early monitoring methods of $\mathrm{SF}_{6}$ gas leakage, which has low monitoring efficiency and may bring potential danger to the monitoring personnel (Yang et al., 2020). Therefore, it is necessary to fully consider the characteristics of $\mathrm{SF}_{6}$ gas and design a more effective monitoring method (Ding et al., 2020; Polyanin, 2020; Sun et al., 2019; Wang et al., 2019). The characteristics of $\mathrm{SF}_{6}$ are described in Table 1.

Table 1. Gas characteristics of $\mathrm{SF}_{6}$

\begin{tabular}{ll}
\hline Nature & Description \\
\hline Density & The density of $\mathrm{SF}_{6}$ is about five times that of air; \\
Appearance and traits & $\mathrm{SF}_{6}$ is a colorless, odorless, odorless and non-toxic non-combustion \\
Melting point & gas under normal conditions; \\
Boiling point & Minus 62 degrees Celsius \\
Relative density & Minus 51 degrees Celsius \\
Relative vapor density & 1.67 \\
Electrical insulation & 5.11 \\
& Under normal pressure, its insulation capacity is more than 2.5 times \\
Arc extinguishing characteristics & that of air \\
& The arc extinguishing capacity is equivalent to 100 times that of air
\end{tabular}


Molecular formula

Molecular weight

Solubility
$\mathrm{SF}_{6}$

146.05

Slightly soluble in water, ethanol and ether, soluble in potassium hydroxide

According to the characteristics of $\mathrm{SF}_{6}$ gas described in Table 1, the $\mathrm{SF}_{6}$ gas adopted in this paper is monitored by spectral absorption method (He et al., 2018b). Based on the strong absorption of $\mathrm{SF}_{6}$ gas to $10.6 \mu \mathrm{m}$ band light, the adopted method is a more advanced $\mathrm{SF}_{6}$ monitoring technology in the world (Gosset et al., 2019). The infrared spectrum of $\mathrm{SF}_{6}$ is shown in Figure 1.

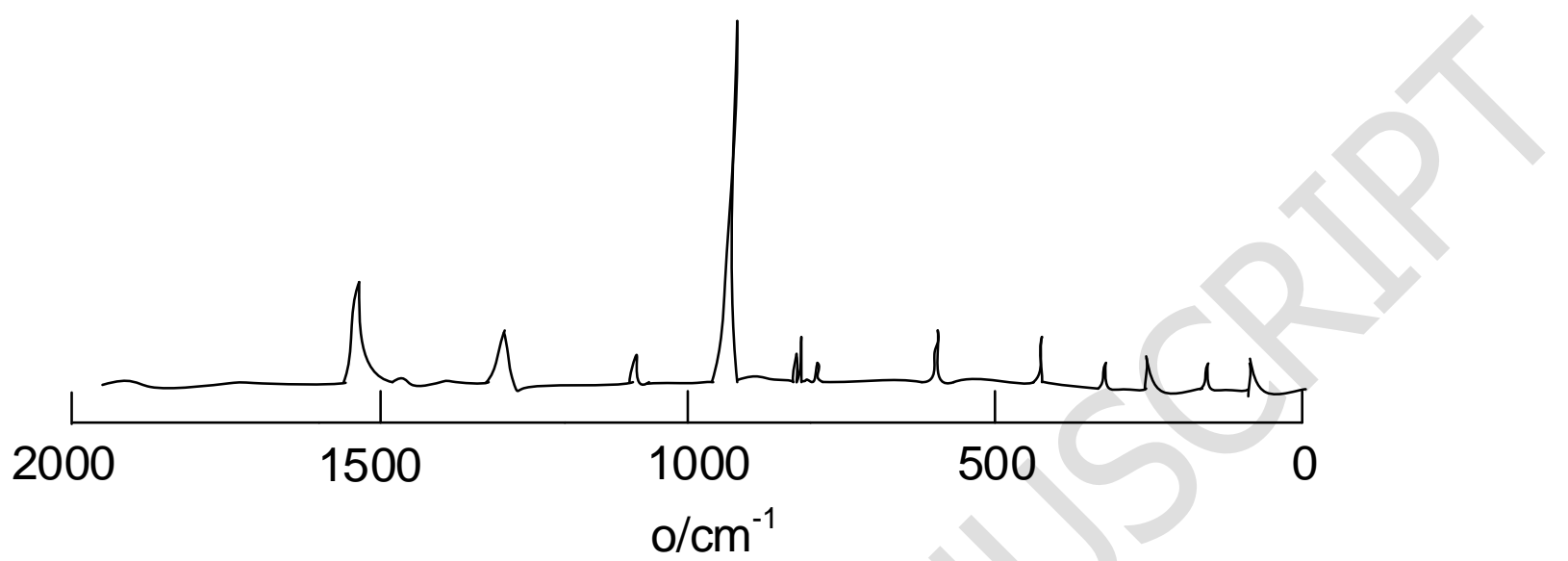

Figure 1. Infrared spectrum of $\mathrm{SF}_{6}$.

$\mathrm{SF}_{6}$ gas has a strong absorption effect on the light of a certain wave band. It can be seen from Figure 1 that the light at the frequency of $950 \mathrm{~cm}^{-1}$ is obviously weakened, and an obvious absorption band of $\mathrm{SF}_{6}$ gas can be seen in this region. According to the relationship between wavelength and frequency, the absorption band of $\mathrm{SF}_{6}$ is about $10.6 \mu \mathrm{m}$.

\subsection{Hardware design of nano sensor system}

According to the characteristics of $\mathrm{SF}_{6}$ gas, the laser is used as the monitoring hardware to monitor the near zero emission state of $\mathrm{SF}_{6}$ gas. The laser used in this paper is the lasy series Lasy-5 laser produced by Datong laser (Mihaita et al., 2019), which has the following characteristics as shown in Table 2.

Table 2. Laser parameters

\begin{tabular}{ll}
\hline Parameter & Numerical value \\
\hline Average power & 5 watts \\
Average power of pulse & 5 watts \\
Bare waist diameter & $2.4 \mathrm{~mm}$ \\
Full angle divergence (milliradians) & Five point five \\
Wavelength (micron) & Ten point six \\
Voltage (V) & DC 28 \\
Working temperature & Less than $90 \%$, non condensing \\
Working pressure & $0.7 \sim 1.3$ Standard atmospheres \\
\hline
\end{tabular}

The laser wavelength generated by Lasy-5 laser is $10.6 \mu \mathrm{m}$, which is just within the strongest absorption band of $\mathrm{SF}_{6}$ gas (Daniell et al., 2019; Hu et al., 2020; Li et al., 2018; Zhang et al, 2020). Therefore, Lasy-5 laser can meet the requirements of this monitoring design.

\subsection{Realization of nano sensor for near zero emission monitoring of $S F_{6}$}

\subsubsection{Working Principle of Nanosensor}

Nanosensors have been widely used in the fields of biology, chemistry, machinery, aviation, military and so on. Compared with traditional sensors, they have smaller size and improved accuracy. Monitoring gases by 
using nanomaterial catalytic luminescence sensors is a typical example of the application of nanomaterials (Tamersit, 2019). In the process of luminescence, the nanomaterial catalytic luminescence sensor mainly consumes oxygen in the air and the monitored gas molecules, and the consumption of solid catalyst is very small. Therefore, as an organic gas sensor, the nanosensor is expected to become a new type of chemiluminescence gas sensor with practical value, which overcomes the shortcomings of traditional chemiluminescence sensor (Pour et al., 2019). Catalytic luminescence signal monitoring can provide rich identification information on the intensity, wavelength and other aspects of monitored objects, and can realize real-time monitoring of the substances with low luminous intensity. The principle of the sensor is shown in Figure 2.

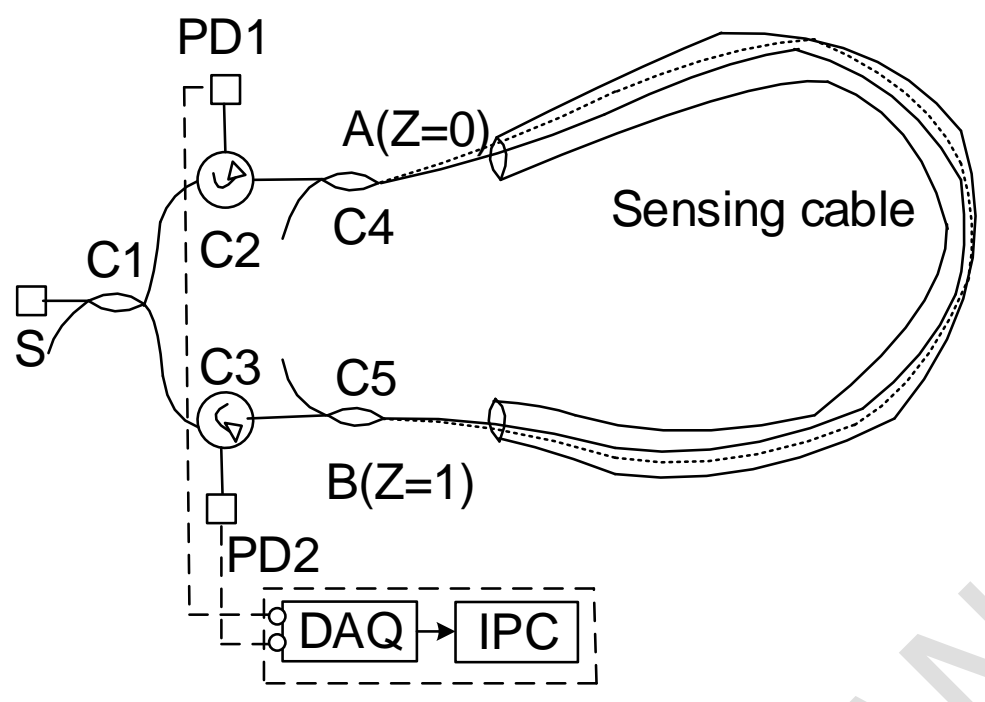

Figure 2. Working principle diagram of cataluminescence sensor.

According to Figure 2, when the monitored gas is introduced to the surface of nanomaterials, the catalytic luminescence reaction will occur on the surface of each sensor unit, and the sensor array will record the luminous intensity of each unit.

\subsubsection{Design of nano sensor array}

According to the working principle of the nano sensor, when the organic gas contacts the surface of nanomaterials, chemiluminescence will occur, and its signal will produce different properties of the monitored organic gas and nanomaterials. However, the sensor made of a single nanomaterial can only monitor a certain organic gas, if a variety of different nanomaterials are as sensing units to make sensor arrays, different organic gases can be monitored (El Khalidi et al., 2019; Lv and Kumar, 2020; Song et al., 2020; Yan et al., 2019). Therefore, before monitoring the near zero emission of $\mathrm{SF}_{6}$, the sensor array should be designed based on the catalytic luminescence of nanomaterials. Figure 3 shows the layout of nanomaterials in the nano sensor array (Cordao Neto et al., 2018).

It can be seen from Figure 3 that a $3 \times 2$ nano sensor array is composed of nanomaterials $\left(\mathrm{SiO}_{2} / \mathrm{Fe}_{2} \mathrm{O}_{3}, \mathrm{Fe}_{2} \mathrm{O}_{3}\right.$, $\mathrm{ZrO}_{2}, \mathrm{CeO}_{2}, \mathrm{Y}_{2} \mathrm{O}_{3}, \mathrm{TiO}_{2}$ ) in the designed order, and the area of each unit is about $8 \times 12 \mathrm{~mm}^{2}$. 


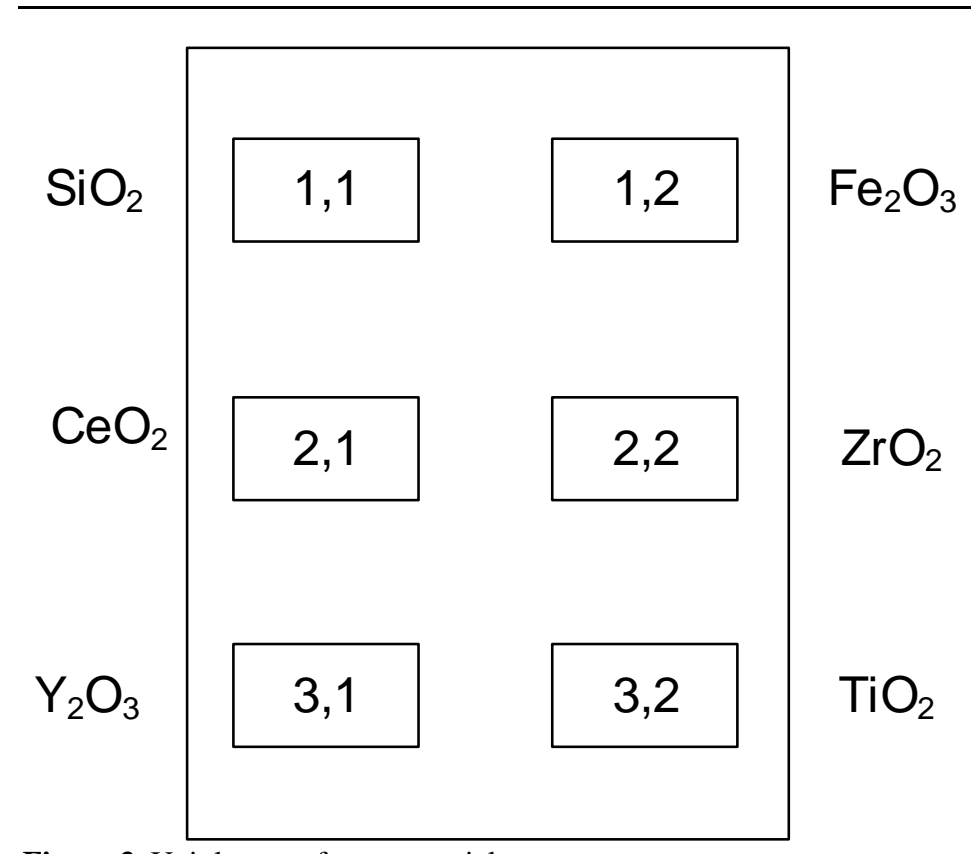

Figure 3. Unit layout of nanomaterials

Considering that the luminous intensity of $\mathrm{SF}_{6}$ on different nano materials is different, it is necessary to set the luminous intensity threshold of each unit separately, and the error of the data measured by the instrument should be considered. Through studying the luminous intensity of gas on six kinds of materials, the threshold of each unit is set, as shown in Figure 4.

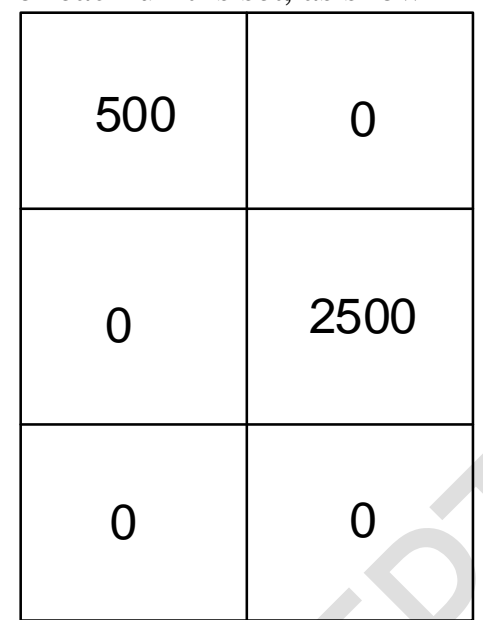

Figure 4. Threshold setting of sensor unit.

\subsubsection{Nanosensor for near zero emission monitoring of $S F_{6}$}

According to the working principle of the nanosensor and the design results of the sensor array, $F_{1} \sim F_{6}$ is set as the anchor node, which is close to the unknown sensor node. The unknown sensor node is set as $K_{i}$. According to the maximum likelihood estimation principle, at least three anchor nodes are selected for $K_{i}$ to estimate its location information.

Taking anchor nodes $F_{1}, F_{2}$, and $F_{3}$ as an example, an inner angle $\beta$ in the array structure needs to meet the following conditions:

$$
\cos \beta=\left[\frac{\left(F_{1} F_{2}\right)^{2}+\left(F_{1} F_{3}\right)^{2}-\left(F_{2} F_{3}\right)^{2}}{2\left(F_{1} F_{2}\right) \cdot\left(F_{2} F_{3}\right)}\right]
$$

Where $F_{1} F_{2},\left(F_{2} F_{3}\right),\left(F_{1} F_{3}\right)$ are the distances between anchor nodes, respectively.

The collinearity of the anchor node in the near-zero emission state monitoring nano sensor of $\mathrm{SF}_{6}$ gas can be defined as: 


$$
\zeta=\max _{i} \chi_{i} \quad i=1,2,3,4,5,6
$$

Where $\chi_{i}$ represents the inner corner of a triangle formed by using 6 anchor nodes in the monitoring sensor for near-zero emission of $\mathrm{SF}_{6}$ gas. When formula (2) satisfies the following conditions, it indicates that the sensor nodes are fully collinear.

$$
\pi / 6 \leq \zeta \leq \pi
$$

According to formula (3), when the collinearity of the anchor node is $\zeta=\pi / 6 \pi$, the monitoring error of the gas near zero emission state of $\mathrm{SF}_{6}$ is the smallest. Set the sensor node distance to $d(0<d<0.6)$. After the anchor node information is received, we calculate the collinearity of multiple anchor nodes that are closer to the unknown sensor node according to formula (2). When formula (4) is met, the trilateration method is adopted to solve the position information of unknown sensor nodes. Or, an anchor node closer to the unknown sensor node $K_{i}$ is selected to replace the candidate anchor node until the following formula (4) is met:

$$
0 \leq|\cos \zeta| \leq d
$$

It is assumed that the sensor node for near zero emission state monitoring of $\mathrm{SF}_{6}$ gas is constructed by the node with unknown position (i.e. the unknown node for near zero emission state monitoring of $\mathrm{SF}_{6}$ gas) and the sensor layer node with known position (i.e. the sensor anchor node for near zero emission state monitoring of $\mathrm{SF}_{6}$ gas) (Mansouri et al., 2019; Subramanian et al., 2019). Let $p$ represent the number of monitoring nodes and $q$ represent the number of anchor nodes, the parameters of the monitoring nodes in the two-dimensional plane are:

$$
I=\left|\sum_{p=1}^{q} X^{0}(p+1)-X^{0}(0+1)+0.5\left[X^{0}(q+1)-X^{0}(0+1)\right]\right|
$$

The position parameter of the nano sensor node for near-zero emission monitoring of $\mathrm{SF}_{6}$ gas is $D_{(p, q)}$, which can be expressed as follows:

$$
D_{(p, q)}=\left[\varpi_{N}, \varpi_{M}\right]^{G}
$$

Where $\varpi_{N}$ and $\varpi_{M}$ respectively represent the position parameters of the sensor node and the error anchor node of the near-zero emission state of $\mathrm{SF}_{6}$ gas; $T$ represents the limiting threshold.

Set $X(n)$ as the neighbor node of node $x$, and $Y(n)$ as the set of neighbor nodes. The calculation expression is:

$$
Y(n)=\sum_{x=1}^{q} x(i, j) \quad k=1,2, . ., n
$$

Where $(i, j)$ represents a pair of adjacent nodes.

Assuming that the adjacent nano sensor nodes for the near-zero emission monitoring of $\mathrm{SF}_{6}$ gas are consistent (Magne et al., 2020; Sun et al., 2020), there is a relative measurement relationship between node $i$ and node $j$ in the nano sensor for the near-zero emission state monitoring of $\mathrm{SF}_{6}$ gas, namely:

$$
x(i, j)=1
$$

If there is no relative measurement relationship between node ${ }^{i}$ and node $j$ in the nano sensor for near-zero emission monitoring of $\mathrm{SF}_{6}$ gas, then:

$$
x(i, j)=0
$$

The relative observation information in the nano sensor node of the near-zero emission state monitoring of $\mathrm{SF}_{6}$ gas includes distance, angle, range, etc. Among them, the distance information is the most accurate, so the relative observation between nodes is selected as the distance observation $D_{i, j}$, which can be expressed as follows:

$$
D_{i, j}=h_{i, j}+l_{i, j}
$$

Where $h_{i, j}$ represents the measurement error of the true distance between the nano sensor node ${ }^{i}$ and the node $j ; l_{i, j}$ represents the true distance between the nano sensor node $i$ and the node $j$, and the calculation expression is as follows: 


$$
l_{i, j}=\sqrt{\left[\left(o_{i}-o_{j}\right)^{2}+\left(c_{i}-c_{j}\right)^{2}\right] \times\left(o_{i} c_{i}+o_{j} c_{j}\right)}
$$

Where $\left(o_{i}, o_{j}\right)$ and $\left(c_{i}, c_{j}\right)$ represent the coordinates in the nano sensor array of nano sensor node $i$ and node $j$, respectively.

Assuming that the set of distance observations obtained by the nano sensor for the near-zero emission monitoring of $\mathrm{SF}_{6}$ gas is $D_{i j}=\left\{d_{1}, d_{2}, \ldots, d_{n}\right\}$, the specific expression is as follows:

$$
D_{i j}=\left(\frac{\|i, j\|^{2}}{d^{2}}\right) \times v^{k}
$$

Where $v^{k}$ represents the node series observation value.

Then the monitoring joint probability of the nano sensor for the near-zero emission monitoring of $\mathrm{SF}_{6}$ gas is:

$$
E=\sum_{i=1, j=1}^{n} \frac{\tau_{i, j} \cdot C(i, j)}{\tau_{i, j} \cdot D_{i j}}
$$

Where $\tau_{i, j}$ represents the location parameters of nano sensor node $i$ and node $j ; C(i, j)$ represents the relative observation prior probability between nodes in the nano sensor.

Assuming that the nano sensor anchor node for monitoring near-zero emission state of $\mathrm{SF}_{6}$ gas has certain online monitoring prior information at the initial moment:

$$
\psi_{i}=\left\{\left[w \varphi\left(x_{i}\right)-b\right]\right\} \times \varepsilon+\xi_{i}
$$

Where $\psi_{i}$ represents the prior probability of the nano sensor node $i ; \varphi$ represents the variation coefficient of the reliability coverage of the nano sensor network monitoring area; $b$ represents the position error parameter of the nano sensor anchor node.

In the traditional method of using sensor nodes to monitor the near zero emission state of SF6 gas, the location parameters of anchor nodes are not accurate enough, so it is necessary to apply nano sensors in the near zero emission state monitoring of SF6 gas. The stability of nano sensor is considered in the design of emission condition monitoring, so the design of nano sensor for near zero emission condition monitoring of sulfur hexafluoride is completed.

\section{Results and Discussion}

In order to verify the applicability and effectiveness of the proposed nano sensor for near zero emission monitoring of $\mathrm{SF}_{6}$, relevant experimental tests were designed.In the experiment, in order to highlight the advantages of the proposed method, the ultraviolet imaging remote sensing monitoring method and the acoustic method are comparatively analyzed, so as to ensure the reliability of the experimental results ( $\mathrm{Li}$ and Zhang, 2020; Wen et al., 2019; Xiao et al., 2019).

\subsection{Preparation for the Experiment}

Before the experiment, we need to set the experimental environment. In 2008, Institute of Atmospheric Physics, Chinese Academy of Sciences completed and improved the atmospheric environment real-time monitoring network, which includes 17 ground stations and 6 sub stations on the tower. Eight stations in the monitoring

\begin{tabular}{|c|c|c|c|}
\hline Sampling point & Surrounding conditions & Sampling time & Number of valid samples \\
\hline 1 & $\begin{array}{l}\text { It is close to urban roads and } \\
\text { residential areas }\end{array}$ & 2019.5.26 & 102 \\
\hline 2 & $\begin{array}{l}\text { Keep away from roads and } \\
\text { residential areas }\end{array}$ & 2019.6.10 & 109 \\
\hline 3 & $\begin{array}{l}\text { It is close to the road and far from } \\
\text { the residential area }\end{array}$ & 2019.7.26 & 87 \\
\hline
\end{tabular}
network are selected to collect $\mathrm{SF}_{6}$ emission samples. The specific location, surrounding conditions, observation time and effective sample number of each sampling point are shown in Table 3.

Table 3. Sampling points 


\begin{tabular}{llll}
\hline 4 & & & \\
\hline & $\begin{array}{l}\text { It is close to urban roads and } \\
\text { residential areas }\end{array}$ & 2019.8 .01 & 135 \\
5 & $\begin{array}{l}\text { It is far away from urban roads } \\
\text { and residential areas }\end{array}$ & 2019.9 .13 & 149 \\
6 & Near site & 2019.10 .21 & 87 \\
7 & Close to school & 2019.12 .06 & 54 \\
\hline
\end{tabular}

Taking the selected sampling points as the basic test environment, the historical data of $\mathrm{SF}_{6}$ emission monitoring in the whole year of 2019 is obtained, as shown in Figure 5.

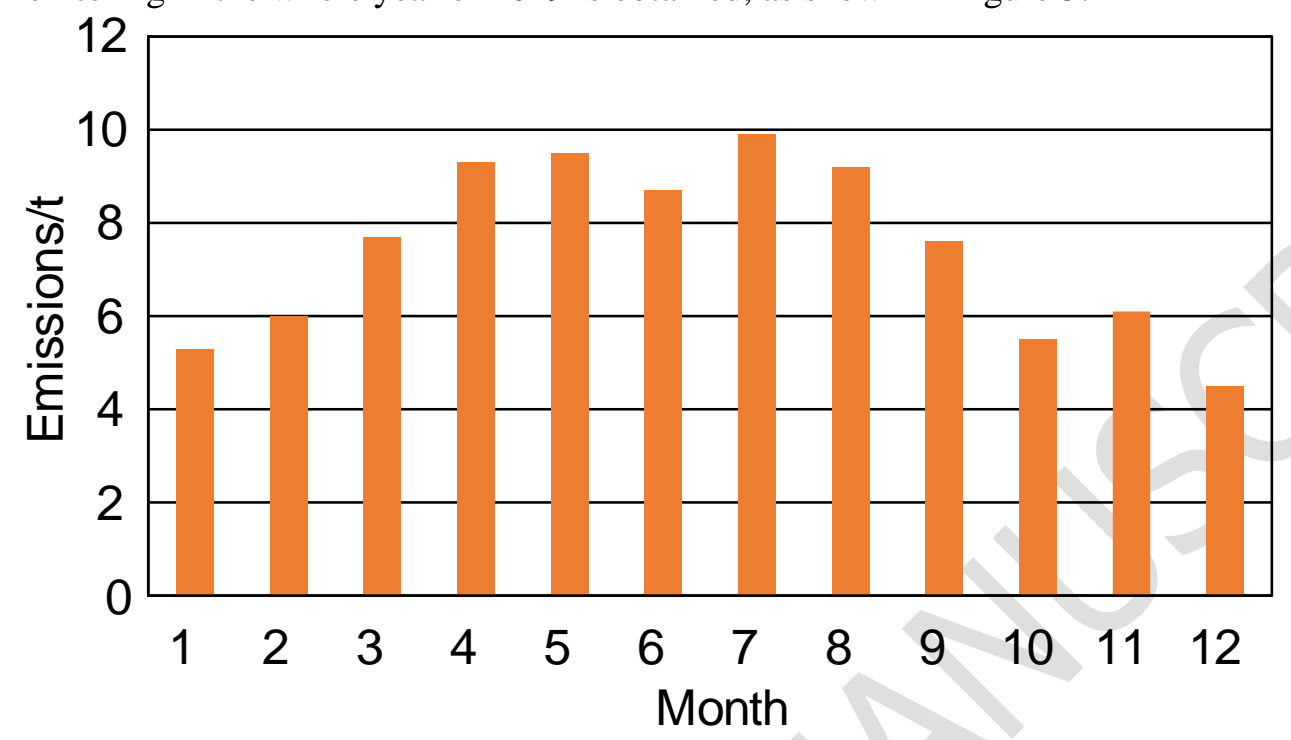

Figure 5. Historical data of $\mathrm{SF}_{6}$ emission monitoring.

On the basis of the above experimental environment, MATLAB software is used to process the monitoring data. In order to ensure the accuracy of the later experimental results, it is necessary to maintain the consistency of the experimental conditions.

\subsection{Analysis of Experimental Results}

\subsubsection{Comparison of Monitoring Accuracy}

In the above research, the near zero emission of $\mathrm{SF}_{6}$ was monitored by nano sensor. In order to prove the effectiveness of the proposed method, different methods are tested by taking the monitoring accuracy as the experimental index, and the results are shown in Table 4.

Table 4. Comparison of monitoring accuracy of different methods

\begin{tabular}{llll}
\hline \multirow{2}{*}{$\begin{array}{l}\text { Experiment } \\
\text { times/time }\end{array}$} & Monitoring accuracy/\% & & \\
\cline { 2 - 4 } & The proposed method & $\begin{array}{l}\text { Ultraviolet imaging remote sensing } \\
\text { monitoring method }\end{array}$ & Sonic method \\
\hline 10 & 81.56 & 76.15 & 73.28 \\
20 & 88.21 & 77.93 & 73.56 \\
30 & 90.43 & 78.40 & 74.21 \\
40 & 91.12 & 78.59 & 74.59 \\
50 & 91.17 & 79.85 & 75.02 \\
60 & 92.25 & 80.16 & 75.67 \\
70 & 91.36 & 80.26 & 76.04 \\
80 & 92.03 & 81.45 & 76.29 \\
90 & 92.94 & 81.59 & 77.06 \\
100 & 92.99 & 82.37 & 78.93 \\
\hline
\end{tabular}


According to the analysis of the data in Table 4, when the number of experiments is 50, the monitoring accuracy of the designed nano sensor method is $91.17 \%, 79.85 \%$ and $75.02 \%$, respectively; When the number of experiments is 100 , the monitoring accuracy of the proposed method, the ultraviolet imaging remote sensing method, and the acoustic method is $92.99 \%, 82.37 \%$ and $78.93 \%$, respectively. The results show that the monitoring accuracy of the nano sensor method is significantly higher than that of the other two methods, indicating that the monitoring results of the nano sensor method are more reliable, which can provide reliable data basis for the study of gas emission and atmospheric environment monitoring.

\subsubsection{Comparison of Monitoring Time}

In order to prove the effectiveness of the proposed method, different methods are tested with the monitoring time as the experimental index, and the results are shown in Figure 6.

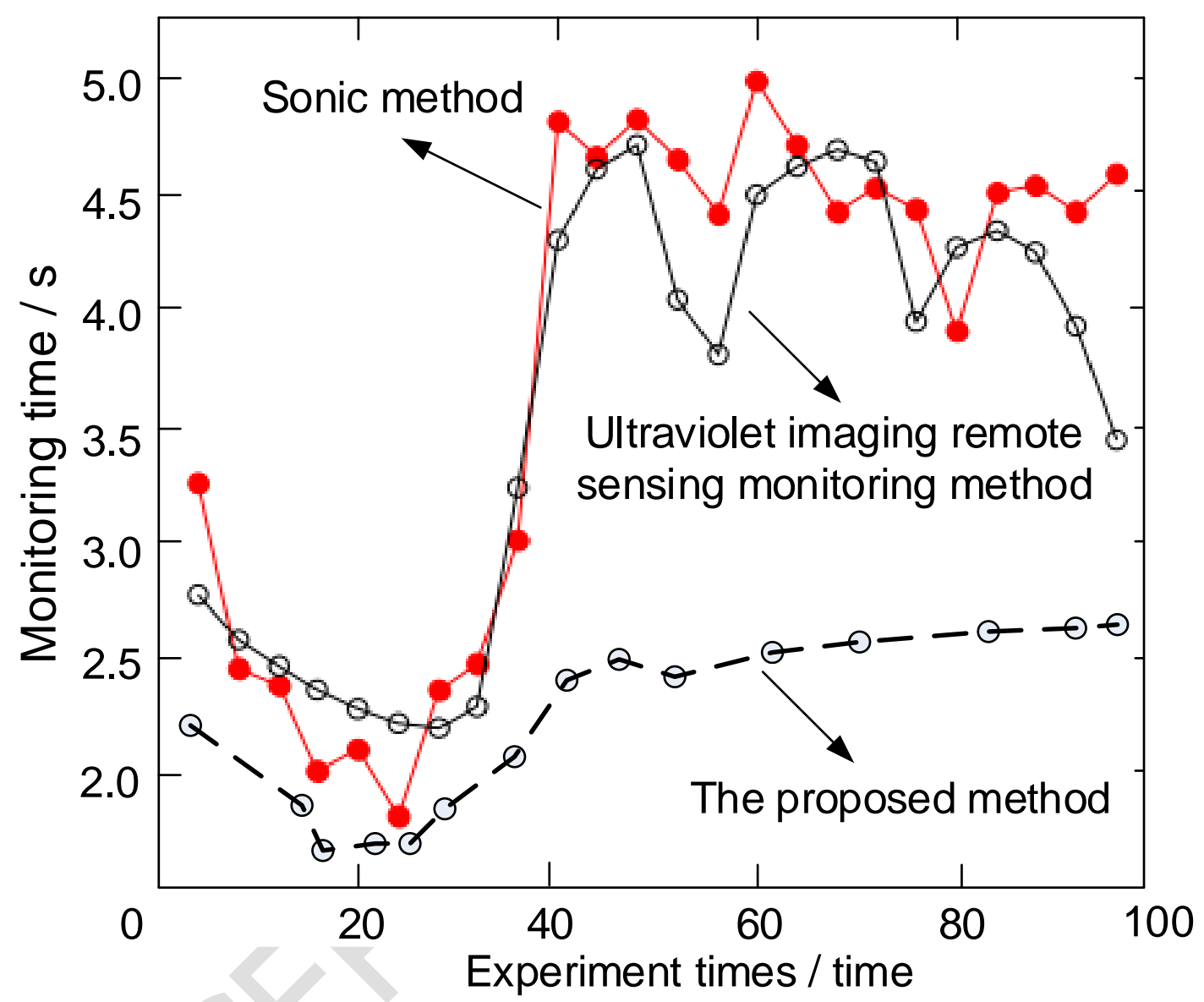

Figure 6. Comparison of monitoring time of different methods.

It can be seen from the analysis in Figure 6 that the monitoring time of the proposed nano sensor method is always lower than that of the ultraviolet imaging remote sensing method and the acoustic method.With the increase of the number of experiments, the monitoring time of the proposed method does not exceed $3.0 \mathrm{~s}$, the maximum monitoring time of the ultraviolet imaging remote sensing method is more than $4.5 \mathrm{~s}$, and the maximum monitoring time of the acoustic method is close to $5.0 \mathrm{~s}$. The comparison shows that the proposed method has higher monitoring efficiency. This is due to that the proposed method uses nanosensor to monitor the near zero emission state of $\mathrm{SF}_{6}$. The proposed method effectively overcomes the shortcomings of traditional chemiluminescence sensor which easily causes reagent consumption and shedding, thus reducing the influence of multiple factors on the monitoring process and improving the monitoring efficiency.

\subsubsection{Monitoring Sensitivity}

In order to further test the application performance of the nano sensor method, the performances of different methods in monitoring near zero emission of $\mathrm{SF}_{6}$ are comparatively analyzed by taking the monitoring sensitivity is used as the evaluation index. 
The monitoring sensitivity is denoted by $\delta_{i}$, and the calculation formula is as follows:

$$
\eta=\frac{S_{\eta}}{S_{\varepsilon}} \times t_{i}
$$

Where $S_{\eta}$ represents the reflected light from the monitoring sampling point; $S_{\varepsilon}$ represents the distance between different monitoring sampling points; $t_{i}$ represents the number of monitoring.

The comparison results of monitoring sensitivity of different methods are shown in Figure 7.

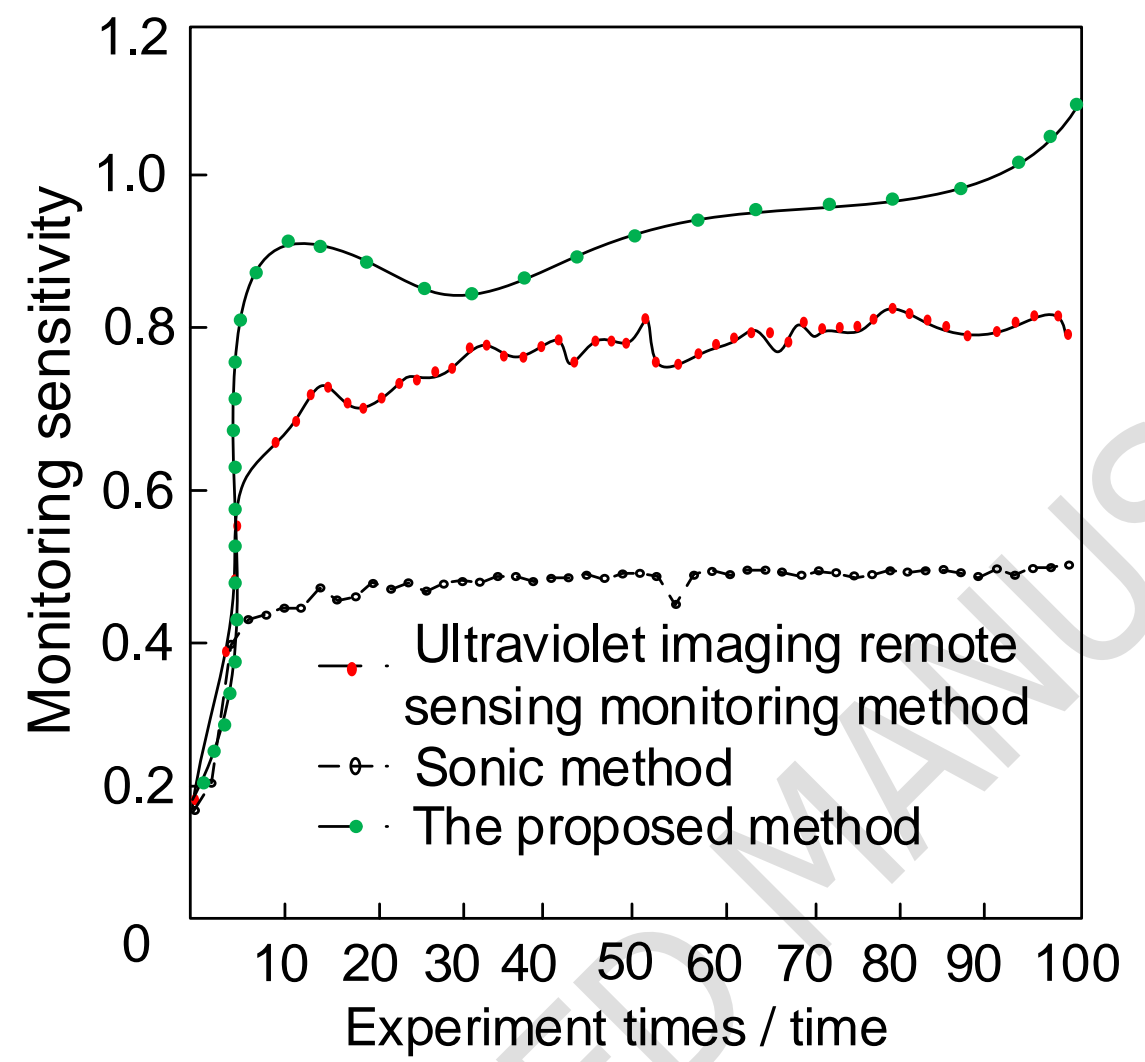

Figure 7. Comparison of monitoring sensitivity of different methods.

It can be seen from the analysis of Figure 7 that with the increase of the number of experiments, the monitoring time of different methods does not show a regular change trend, but on the whole, the monitoring sensitivity of the proposed method is significantly higher than that of the ultraviolet imaging remote sensing monitoring method and the acoustic method, indicating a better monitoring effect of proposed method.

In conclusion, the nano sensor method is superior to the traditional method in terms of monitoring time, monitoring sensitivity and monitoring accuracy, which fully verifies the practical application value of the nano sensor method.

\section{Conclusion}

In recent years, studies have shown that the concentration of $\mathrm{SF}_{6}$ in the atmosphere is increasing rapidly at the rate of $7 \% \sim 8 \% . \mathrm{SF}_{6}$ in the atmosphere is mainly emitted by human activities, of which $80 \%$ comes from the insulators and high-voltage converters of electrical equipment, and the remaining $20 \%$ is mainly released from magnesium and aluminum industries (Okolie et al., 2019). The impact of $\mathrm{SF}_{6}$ on the atmospheric environment is mainly manifested by the greenhouse effect and the destruction of the ozone layer. At present, the issue of $\mathrm{SF}_{6}$ emission and control has received attention from all walks of life. Under the above background, this paper proposes Research on nano sensor for near zero emission monitoring of $\mathrm{SF}_{6}$. The main research results are concluded as follows:

- In this work, we analyzed the characteristics of $\mathrm{SF}_{6}$ gas, and used the spectroscopic absorption method to conduct preliminary monitoring of $\mathrm{SF}_{6}$ gas emissions based on the analysis results. 
- The lasy series Lasy-5 laser is used as the $\mathrm{SF}_{6}$ gas monitoring hardware. The laser wavelength generated by the laser is just within the strongest absorption band of the $\mathrm{SF}_{6}$ gas, which can meet the requirements of monitoring.

- The sensor array is designed according to the working principle of the nano sensor, and finally the monitoring of the near-zero emission of $\mathrm{SF}_{6}$ is realized through the optimization of the nano sensor nodes.

- The experimental results show that the nano sensor method has significant advantages in monitoring accuracy, monitoring time and monitoring sensitivity, indicating that the method can be applied to gas emission monitoring, with better monitoring effect being achieved.

\section{References}

Blanco-Rayon, E., Guilhermino, L., Marigomez, I. and Izagirre, U., 2019. Collection and transport of sentinel mussels in biomarker-based coastal pollution monitoring: current flaws and reliable practices. Ecological Indicators, 103(8), 722-734.

Cordao Neto, M.P., Hernandez, O., Reinaldo, R.L., Borges, C. and Caicedo, B., 2018. Study of the relationship between the hydromechanical soil behavior and microstructure of a structured soil. Earth Sciences Research Journal, 22(2), 91-101.

Daniell, A., Malo, D.S. and Van Deventer, P.W., 2019. Monitoring the pollution effects from a gold tailing storage facility on adjacent land through landscape function analysis. Environmental Earth Sciences, 78(3), e82(1)-e82(14).

Ding, P., Xu, D., Dong, N., Chen, Y., Xu, P., Zheng, D. and Li, X., 2020. A high-sensitivity $\mathrm{H}_{2} \mathrm{~S}$ gas sensor based on optimized $\mathrm{ZnO}-\mathrm{ZnS}$ nano-heterojunction sensing material. Chinese Chemical Letters, 31(8), 2050-2054.

El Khalidi, Z., Hartiti, B., Siadat, M., Comini, E., Arachchige, H.M.M.M., Fadili, S. and Thevenin, P., 2019. Acetone sensor based on $\mathrm{Ni}$ doped $\mathrm{ZnO}$ nanostructues: growth and sensing capability. Journal of Materials Science, 30(8), 7681-7690.

Garmendia, M., Silvia, F.O.D.V., Linero, O., Gredilla, A., Arana, G., Soto, M. and Diego, A.D., 2019. Long term monitoring of metal pollution in sediments as a tool to investigate the effects of engineering works in estuaries. A case study, the Nerbioi-ibaizabal estuary (Bilbao, Basque Country). Marine Pollution Bulletin, 145(8), 555-563.

Gosset, A., Durrieu, C., Barbe, P., Bazin, C. and Bayard, R., 2019. Microalgal whole-cell biomarkers as sensitive tools for fast toxicity and pollution monitoring of urban wet weather discharges. Chemosphere, 217(2), 522-533.

Grace, R.K. and Manju, S., 2019. A comprehensive review of wireless sensor networks based air pollution monitoring systems. Wireless Personal Communications, 108(4), 2499-2515.

He, L., Chen, Y. and Li, J., 2018. A three-level framework for balancing the tradeoffs among the energy, water, and air-emission implications within the life-cycle shale gas supply chains. Resources, Conservation and Recycling, 133, 206-228.

He, L., Shen, J. and Zhang, Y., 2018. Ecological vulnerability assessment for ecological conservation and environmental management. Journal of Environmental Management, 206, 1115-1125.

Hu, J., Zheng, B., Wang, C., Zhao, C., Hou, X., Pan, Q. and Xu, Z., 2020. A survey on multi-sensor fusion based obstacle detection for intelligent ground vehicles in off-road environments. Frontiers of Information Technology \& Electronic Engineering, 21(5), 675-692.

Li Sun, L., Li, C., Zhang, C.W., Su, Z.X. and Chen, C., 2018. Early monitoring of rebar corrosion evolution based on FBG sensor. International Journal of Structural Stability and Dynamics, 18(08), 18400011842018.

Li, P. and Zhang, Z.K., 2020. Self-powered 2D material-based pH sensor and photodetector driven by monolayer mose2 piezoelectric nanogenerator. ACS Applied Materials \& Interfaces, 12(52), 5813258139.

Lv, Z. and Kumar, N., 2020. Software defined solutions for sensors in 6G/IoE. Computer Communications, 153, $42-47$.

Magne, S., Nehr, S., Buet, X., Bentab, A., Porcheron, E., Grosseuvres, R., Studer, E., Scarpa, R., Abdo, D. and Widloecher, J.L., 2020. In situ gas monitoring by fiber-coupled Raman spectrometry for h-risk 
management in nuclear containment during a severe nuclear accident. IEEE Transactions on Nuclear Science, 67(4), 617-624.

Mansouri, E., Karamdel, J., Ahmadi, M.T. and Berahman, M., 2019. Analytical modeling of phosphorenebased $\mathrm{NO}_{2}$ gas sensor. International Journal of Modern Physics B, 33(14), e1950143(1)-e1950143(10).

Marchant, C., Leiva, V., Christakos, G. and Cavieres, M.F., 2019. Monitoring urban environmental pollution by bivariate control charts: New methodology and case study in Santiago, Chile. Environmetrics, 30(5), $1-17$.

Mihaita, A.S., Dupont, L., Chery, O., Camargo, M. and Cai, C., 2019. Evaluating air quality by combining stationary, smart mobile pollution monitoring and data-driven modelling. Journal of Cleaner Production, 221(6), 398-418.

Mishra, S., Kushwaha, A., Aggrawal, D. and Gupta, A., 2019. Comparative emission study by real-time congestion monitoring for stable pollution policy on temporal and meso-spatial regions in Delhi. Journal of Cleaner Production, 224(7), 465-478.

Morscher, G.N. and Maxwell, R., 2019. Monitoring tensile fatigue crack growth and fiber failure around a notch in laminate SIC/SIC composites utilizing acoustic emission, electrical resistance, and digital image correlation. Journal of the European Ceramic Society, 39(2-3), 229-239.

Okolie, C.U., Chen, H.M., Zhao, Y.X., Tian, D. and Zhang, L., 2019. Cadmium immobilization in aqueous solution by Aspergillus niger and geological fluorapatite. Environmental Science and Pollution Research, 27(7), 7647-7656.

Polyanin, V.O., 2020. Conceptual approaches to monitoring diffuse pollution of water bodies. Water Resources, 47(5), 785-793.

Pour, G.B., Aval, L.F. and Esmaili, P., 2019. Performance of gas nanosensor in 1-4 per cent hydrogen concentration. Sensor Review, 39(4), 622-628.

Sekhar, P.K., Graf, D., Ojelere, O., Saha, T.K. and Mathur, S., 2020. Electrochemical gas sensor integrated with vanadium monoxide nanowires for monitoring low concentrations of ammonia emission. Journal of the Electrochemical Society, 167(2), e027548(1)-e27548(6).

Song, Y., Xu, M., Li, Z., He, L., Hu, M., He, L., Zhang, Z. and Du, M., 2020. Ultrasensitive detection of bisphenol a under diverse environments with an electrochemical aptasensor based on multicomponent AgMo heteronanostructure. Sensors and Actuators. B, Chemical, 321, e128527(1)-e128527(11).

Subramanian, M., Dhayabaran, V.V. and Shanmugavadivel, M., 2019. Room temperature fiber optic gas sensor technology based on nanocrystalline $\mathrm{Ba} \_3$ (VO_4)2: Design, spectral and surface science. Materials Research Bulletin, 119(11), e110560(1)-e110560(9).

Sun, L., Su, Z., Xia, Y., Zhang, C.W. and Li, C., 2019. Superwide-range fiber Bragg grating displacement sensor based on an eccentric gear: Principles and experiments. Journal of Aerospace Engineering, 32(1), e04018129(1)-e04018129(6).

Sun, Y.C., Li, P., Chang, S.J. and Shi, F., 2020. EEMD combined with improved penn model for noise reduction of gas leakage signal. Computer Simulation, 37(9), 409-414+455.

Tamersit, K., 2019. An ultra-sensitive gas nanosensor based on asymmetric dual-gate graphene nanoribbon field-effect transistor: proposal and investigation. Journal of Computational Electronics, 18(3), 846-855.

Toma, C., Alexandru, A., Popa, M. and Zamfiroiu, A., 2019. IoT solution for smart cities' pollution monitoring and the security challenges. Sensors, 19(15), e3401(1)-e3401(24).

Wang, M., Hu, M., Liu, J., Guo, C., Peng, D., Jia, Q., He, L., Zhang, Z. and Du, M., 2019. Covalent organic framework-based electrochemical aptasensors for the ultrasensitive detection of antibiotics. Biosensors \& Bioelectronics, 132, 8-16.

Xiao, J., Hu, C. and Ouyang, G., 2019. Impacts of oil implied volatility shocks on stock implied volatility in China: Empirical evidence from a quantile regression approach. Energy Economics, 80(5), 297-309.

Yamamura, H., Ding, Q. and Watanabe, Y., 2019. Solid-phase fluorescence excitation emission matrix for insitu monitoring of membrane fouling during microfiltration using a polyvinylidene fluoride hollow fiber membrane. Water Research, 164(11), e114928(1)-e114928(7).

Yan, N., Song, J., Wang, F., Kan, L., Song, J., Wang, W., Ma, W., Zhang, W. and He, G., 2019. Pyrenoviologen-based fluorescent sensor for detection of picric acid in aqueous solution. Chinese Chemical Letters, 30(11SI), 1984-1988.

Zhang, R., Xia, C., Zhou, X., Guo, Y. and Li, W., 2020. Molybdenum disulfide nanomaterial sensor-based detection of breast cancer microRNA. Materials Express, 10(6), 915-921.

Wen, F., Zhao, Y. and Zhang, M., 2019. Forecasting realized volatility of crude oil futures with equity market uncertainty. Applied Economics, 51(59), 6411-6427. 
Wilde, S., 2019. Meeting report air pollution in megacities: Advances in monitoring and modelling. Weather, 74(6), 226-226.

Yang, C., Gao, F. and Dong, M., 2020. Energy efficiency modeling of integrated energy system in coastal areas. Journal of Coastal Research, 103(sp1), 995.

Zuo, X., Dong, M., Gao, F. and Tian, S., 2020. The modeling of the electric heating and cooling system of the integrated energy system in the coastal area. Journal of Coastal Research, 103(sp1), 1022. 